\title{
Perspectives in Galactic Chemical Evolution studies
}

\author{
N. Prantzos ${ }^{\mathrm{a}}$ IAP]Institut d'Astrophysique de Paris, 98bis Bd Arago, 75014 Paris \\ $\mathrm{a}[$
}

In this review I focus on a few selected topics, where recent theoretical and/or observational progress has been made and important developments are expected in the future. They include: 1) Evolution of isotopic ratios, 2) Mixing processes and dispersion in abundance ratios, 3) Abundance gradients in the Galactic disk (and abundance patterns in the inner Galaxy), 4) The question of primary Nitrogen and 5) Abundance patterns in extragalactic damped Lyman- $\alpha$ systems (DLAs).

\section{INTRODUCTION}

Despite the lack of a reliable model of Galactic Chemical Evolution (GCE) considerable progress has been made in the past few years, due to:

- an impressive amount of observational data concerning abundance ratios either in the Milky Way (Carretta et al. 2002, Truran et al. 2002, Andriefsky et al. 2002, and references therein) or in extragalactic systems (e.g. Prochaska and Wolfe 2002);

- the publication of detailed nucleosynthesis yields (e.g. van den Hoek and Groenewegen 1997 for intermediate mass stars; Woosley and Weaver 1995, Nomoto et al. 1997, Limongi et al. 2000, for massive stars; Iwamoto et al. 1999 for SNIa).

Interpreted in the framework of simple "toy" models of GCE these data offer mainly information about: i) evolutionary timescales of corresponding production sites, ii) physics of those sites, iii) nature of relevant nucleosynthetic processes.

In this, highly biased, review I focus on a few selected topics, where recent theoretical and/or obervational progress has been made and important developments are expected in the future.

\section{EVOLUTION OF Mg ISOTOPIC RATIOS IN THE MILKY WAY}

There are very few cases where observations allow to check models of isotopic abundance evolution in the Galaxy, especially concerning the early (i.e. halo) phase of that evolution. One of these rare cases concerns the $\mathrm{Mg}$ isotopes, which are mainly produced by hydrostatic burning in the carbon and neon shells of massive stars. The production of the neutron-rich isotopes ${ }^{25} \mathrm{Mg}$ and ${ }^{26} \mathrm{Mg}$ is affected by the neutron excess (their yields increase with initial stellar metallicity) while ${ }^{24} \mathrm{Mg}$ is produced as a primary (its yield is independent of metallicity). Thus, the isotopic ratios ${ }^{25} \mathrm{Mg} /{ }^{24} \mathrm{Mg}$ and ${ }^{26} \mathrm{Mg} /{ }^{24} \mathrm{Mg}$ are expected to increase with metallicity (Fig. 1, left panel).

In Fig. 1 (right panel) we compare our results of a detailed GCE model (Goswami 

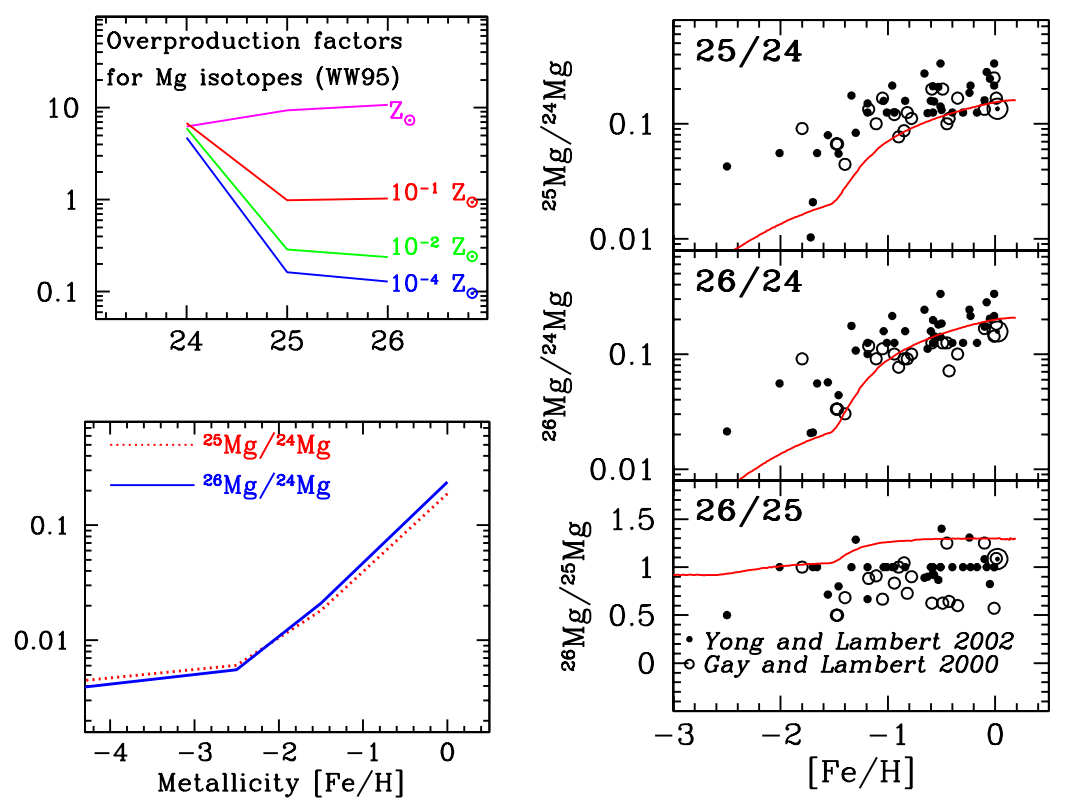

Figure 1. Top left: Overproduction factors of the $\mathrm{Mg}$ isotopes as a function of stellar mass and for various initial metallicities (from WW95). Bottom left: Abundance ratios of the $\mathrm{Mg}$ isotopes (integrated over the Kroupa et al. $1993 \mathrm{IMF}$ ) as a function of initial stellar metallicity. Right: Evolution of Mg isotopic ratios in Milky Ways' halo and local disk stars (from Goswami and Prantzos 2000) with WW95 yields and comparison to observations.

and Prantzos 2000) with observations from various sources, including the yet unpublished data of Yong and Lambert (Yong, private communication). The observational trends are, globally, reproduced by our model, although the model isotopic ratios are systematically lower than observations. This was also noticed in Timmes et al. (1995). It may well be that the Woosley and Weaver (1995, WW95) yields underestimate the importance of the neutron excess in the production of the Mg isotopes or that the Yong and Lambert data systematically overestimate those ratios. Another possibility is that there is some other source of the neutron-rich Mg isotopes in the late halo, like e.g. AGB stars with He-shells hot enough to activate the ${ }^{22} \mathrm{Ne}(\alpha, \mathrm{n})^{25} \mathrm{Mg}$ neutron source. This reaction would provide neutrons for the s-process in those stars, but also produce large amounts of ${ }^{25} \mathrm{Mg}$ and ${ }^{26} \mathrm{Mg}$.

In any case, future observations of isotopic ratios at all metallicities (like those of the Ti isotopes, Young and Lambert in preparation) will bring a substantial refinement in our understanding of stellar nucleosynthesis.

\section{3. (IM)PERFECT MIXING IN THE EARLY GALAXY?}

Standard models of GCE assume instantaneous and perfect mixing of the stellar ejecta with the ISM; this leads to a unique value of metallicity (or of the abundance ratio of 


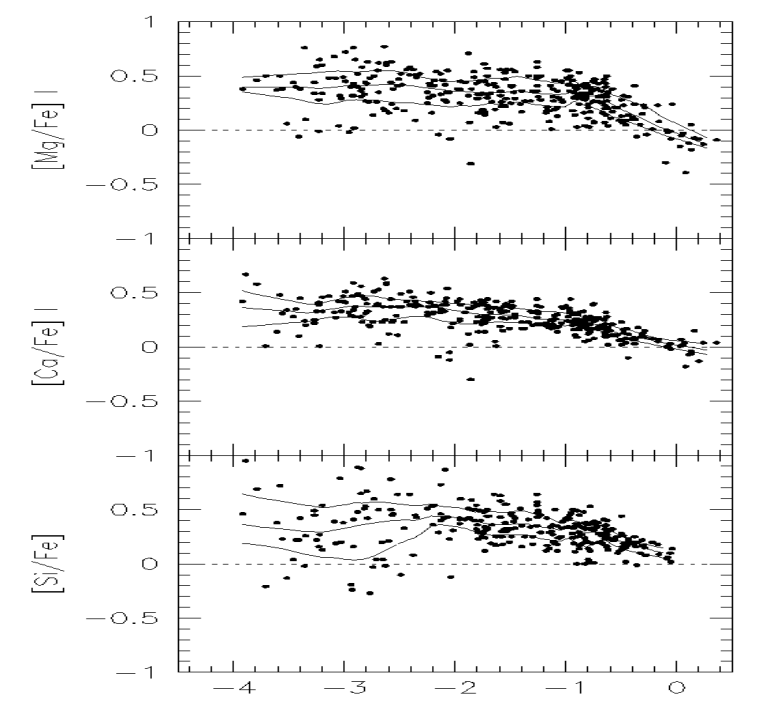

Figure 2. Abundance ratios $[\mathrm{M} / \mathrm{Fe}]$ vs $[\mathrm{Fe} / \mathrm{H}]$ for halo and local disk stars; curves in the middle indicate mean trends, while upper and lower curves indicate $1 \sigma$ scatter (from Carretta et al. 2002). There is no hint for intrsinsic dispersion in the data, if account is taken of the observational uncertainties of $\sim 0.1$ dex.

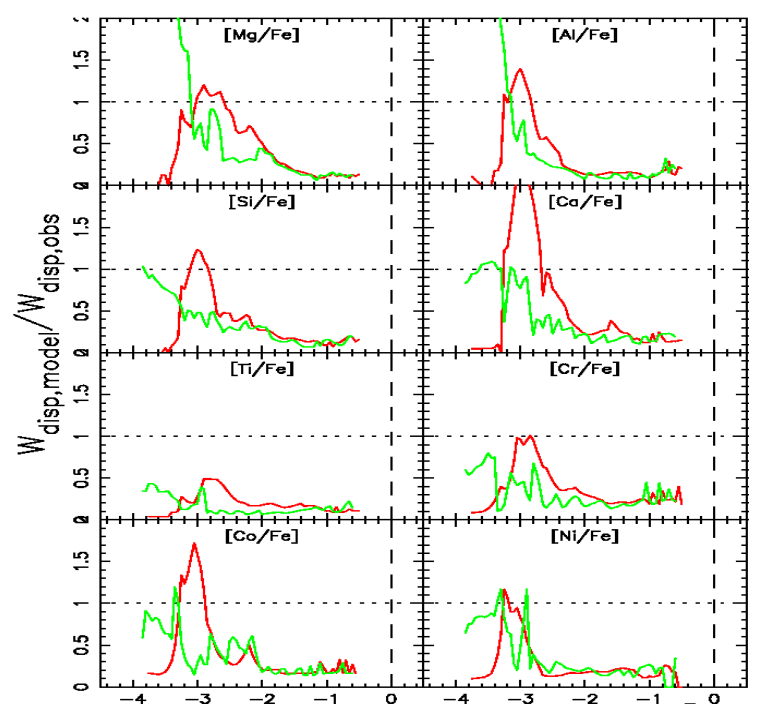

Figure 3. Dispersion (1 $\sigma$ around the mean) of abundance ratios in the solar neighborhood. Model results are obtained with 2 sets of yields: Nomoto et al. 1997 (dark curves) and WW95 (lighter curves) and are divided by corresponding observed scatter as a function of metallicity (from Ishimaru et al. 2002).

any two elements) at all times. This simplistic assumption may not hold, since simple arguments suggest that complete mixing may require many millions of years; during that time a new star may be born with its composition affected mostly by the ejecta of a closeby supernova. A dispersion of abundance ratios, increasing at earlier times (and lower metallicities) is, naively, expected in that case.

Those ideas are supported by observations of s- and r- elements (e.g. Truran et al. 2002). However, for elements up to the Fe-peak Carretta et al. (2002) find no hints for intrinsic dispersion down to $[\mathrm{Fe} / \mathrm{H}] \sim-3$ (Fig. 2). This result is intriguing since simple models, both 1-D (Tsujimoto and Shigeyama 1998; Karlsson and Gustafsson 2001) and 2-D (Argast et al. 2000, 2002), find that an important scatter should be obtained at such low metallicities, its amplitude depending on adopted yields and model assumptions.

The results of recent 1-D models of inhomogeneous GCE (Ishimaru et al. 2002) are displayed in Fig. 3. Two sets of stellar yields are used (Nomoto et al. 1997 and WW95). The resulting dispersion is compared to the observational one (estimated by Ryan et al. 1996). Depending on the adopted set of yields, a rather impotant dispersion is expected for $\alpha$-elements around $[\mathrm{Fe} / \mathrm{H}] \sim-3$ and below, while at metallicities higher than $[\mathrm{Fe} / \mathrm{H}]=-2$ the theoretical scatter is extremely small. Note that uncertainties introduced by observational errors $(\sim 0.1 \mathrm{dex})$ are not taken into account in the displayed model results.

In the future, systematic surveys of low metallicity stars will help to constrain stellar 


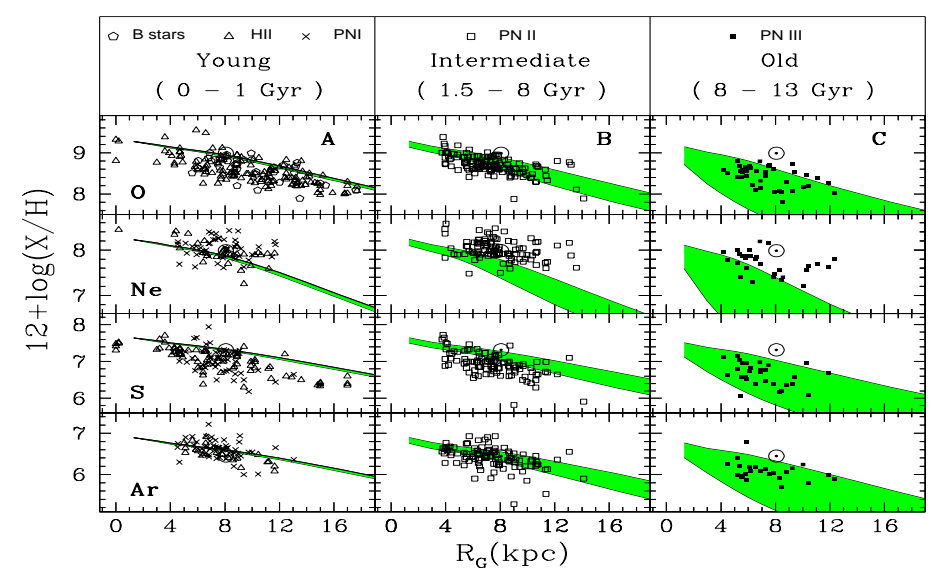

Figure 4. Abundance gradients of $\mathrm{O}, \mathrm{Ne}, \mathrm{S}$ and $\mathrm{Ar}$ in planetary nebulae of various age classes (from left to right) in MW disk; they are compared to model predictions of Hou et al. 2000 (shaded aereas).

yields (and their dependence on stellar mass) and to a better understanding of mixing processes in the ISM.

\section{ABUNDANCES IN THE MILKY WAY DISK}

Observed profiles of MW disk (gas, stars, SFR and abundances) require a larger SF efficiency and/or a smaller timescale of formation (through infall) in the inner disk (Boissier and Prantzos 1999). Models reproducing the above observational constraints with few parameters predict that abundance gradients were steeper in the past (e.g. Hou et al. 2000); they became progressively flatter due to the inside-out formation of the disk (see Fig. 4). That prediction is in agreement with the recent observational results of Maciel and da Costa 2001 (see Fig. 5), based on oxygen abundances of planetary nebulae of various age classes [Note: taking into account the uncertainties in evaluating ages of planetary nebulae, the importance of that agreement should not be overestimated; it is, however, encouraging].

It should be noted that the magnitude of the abundance gradient has been questioned again, recently: Deharveng et al. (2000) and Pilyugin et al. (2002) (based on abundances in HII regions) and Cunha et al. 2002 (based on abundances of B-stars) find systematically smaller oxygen gradients than the "canonical" one of $\sim-0.07 \mathrm{dex} / \mathrm{kpc}$; clearly, the issue of the abundance gradient in the Milky way (and other disk galaxies) is still an open one.

In that respect, it should be noted that a recent study of abundances in Cepheids (with rather well determined distances) finds a bimodal slope of the abundance profile, flatter in the solar neighborhood and steeper inwards (Andrievsky et al. 2002a,b). One of the important points of that work is the determination of stellar abundance patterns concerning a large number of elements (about 25). As can be seen in Fig. 6, there is a well defined abundance pattern, scaled with metallicity, for elements up to the Fe peak 


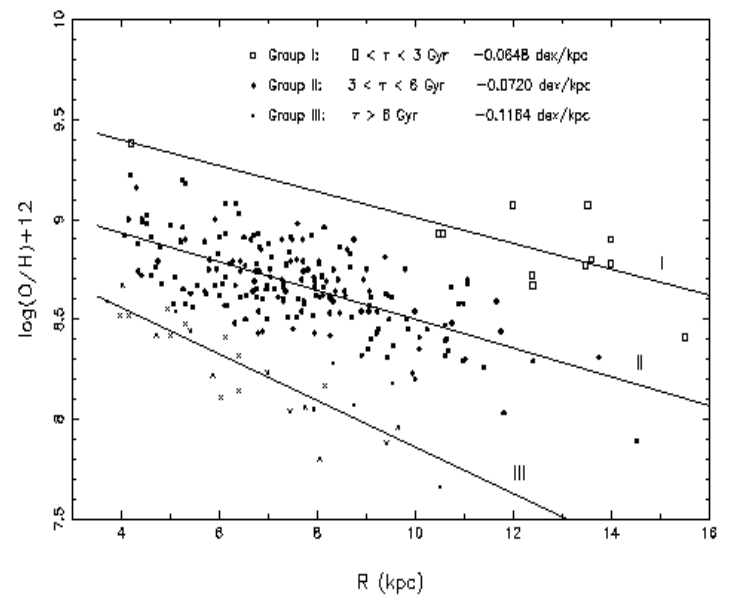

Figure 5. Abundance gradients of $\mathrm{O}$ in MW disk, as traced by planetary nebulae of various age groups, according to Maciel and da Costa (2001).
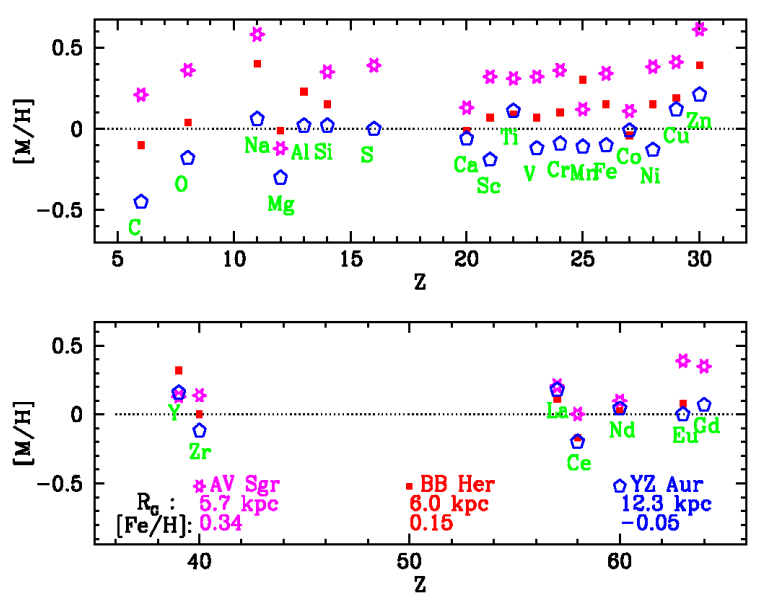

Figure 6. Abundances (compared to solar) of 3 Cepheids in MW disk (symbols in lower panel) from Andrievsky et al. (2002a).

(upper panel), while no such pattern is evident for heavier elements (lower panel). This is the first study establishing detailed abundance patterns in the inner Milky Way; further works of that kind in the future will allow to study abundance patterns in those regions in the same way as we study today detailed abundance patterns (mean trends, scatter, etc) in stars of the galactic halo; interpretation of those patterns will require the calculation of detailed stellar yields up to metallicities of $3 \mathrm{Z}_{\odot}$.

\section{5. "PRIMARY" NITROGEN IN THE EARLY GALAXY?}

Nitrogen is the main product of the CN cycle operating in core H-burning in intermediate and high mass stars, in shell H-burning in stars of all masses and in the putative Hot-Bottom Burning (HBB) in massive (4-8 $\mathrm{M}_{\odot}$ ) AGB stars. In the former two cases $\mathrm{N}$ is produced from the initial $\mathrm{C}$ (and $\mathrm{O}$ ) entering the stars, whereas in the latter from the $\mathrm{C}$ produced by the $3-\alpha$ reaction in the He-shell (and brought to the envelope by convective motions during thermal pulses). Thus, in the first two cases $\mathrm{N}$ is produced as a secondary (its yield being quasi-proportional to initial stellar metallicity) and in the latter as a primary (its yield being quasi-independent of metallicity) [see top panel of Fig. 7].

No stellar evolution model finds $\mathrm{HBB}$ in a self-consistent way up to now: because of uncertainties in the treatment of convection the depth of the convective envelope has to be adjusted in order to reach the required high temperatures (as e.g. in vdHG97). However, there is another way to produce primary $\mathrm{N}$ : rotationally induced mixing may mix protons to He-regions with abundant $\mathrm{C}$ from the $3-\alpha$ reaction. Self-consistent (1-D) models of rotating stars have been recently calculated by Meynet and Maeder (2002, MM02). Their $\mathrm{N}$ yields appear in Fig. 7 (bottom panel): at low metallicities, substantial amounts of quasi-primary $\mathrm{N}$ are produced in intermediate mass rotating stars (with typical rotational 


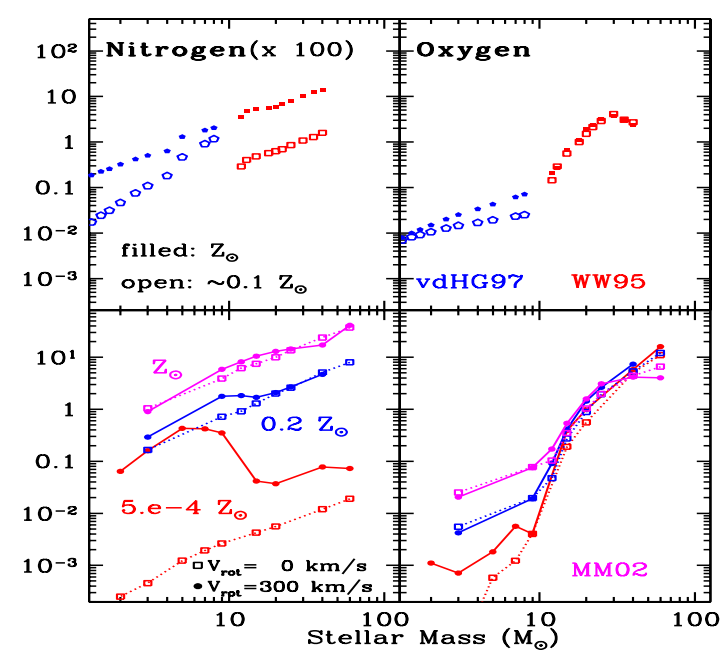

Figure 7. Nitrogen and Oxygen yields. Top: Yields from van den Hoek and Groenevegen 1997 (vdHG97, intermediate mass stars) and WW95 (massive stars). Bottom: Yields from MM02 with rotation (solid curves and no rotation (dotted curves). Yields (total ejected masses) are given for two (upper panel) and three (lower panel) initial metallicities.

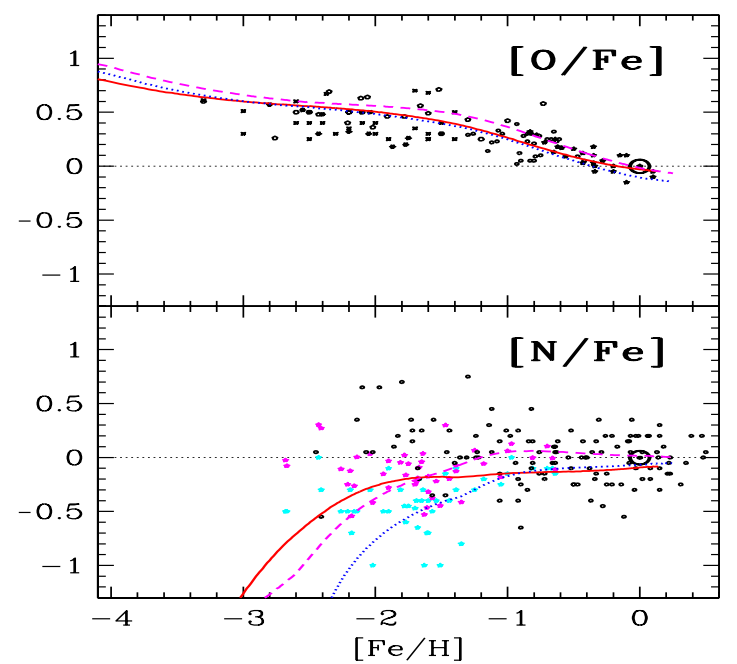

Figure 8. Evolution of $\mathrm{O}$ and $\mathrm{N}$ in solar neighborhood with three sets of stellar yields: vdHG97 + WW95 (solid curve), MM02 with rotation (dashed curves) and MM02 with no rotation (dotted curves). The first two cases, producing primary $\mathrm{N}$ from intermediate mass stars, lead to similar results.

velocities $\mathrm{V}_{R O T}=300 \mathrm{~km} / \mathrm{s}$ ).

The implications of those results for the evolution of $\mathrm{N}$ in the Galaxy are shown in Fig. 8. The $\mathrm{N}$ yields of the rotating stars of MM02 lead to an evolution similar to the one obtained with the HBB yields of vdHG97: N behaves as primary with respect to $\mathrm{Fe}$, starting at $[\mathrm{Fe} / \mathrm{H}] \sim-2$. With "standard" yields of non-rotating stars (with no $\mathrm{HBB}$ ), $\mathrm{N}$ is always produced as secondary, but it behaves as primary w.r.t Fe (its secondary production being matched by the delayed Fe ejection from SNIa) only after $[\mathrm{Fe} / \mathrm{H}] \sim-1$.

Current obervations do not allow to conclude whether primary $\mathrm{N}$ is needed below $[\mathrm{Fe} / \mathrm{H}] \sim-2$. If this were the case, primary $\mathrm{N}$ from massive stars (which dominate at such low metallicities and early times, due to their short lifetimes) would be required; the corresponding mechanism has not been found up to now. Alternatively, the timescales of simple models of GCE (like the one of Fig. 8), which are not seriously constrained by other observations, should have to be revised.

\section{ABUNDANCE PATTERNS IN DLAs}

Damped Lyman $\alpha$ systems (DLAs) are high column density gaseous systems $(\mathrm{N}(\mathrm{HI})>2$ $10^{20} \mathrm{~cm}^{-2}$ ), detected through their absorption lines in the optical spectra of quasars, up 


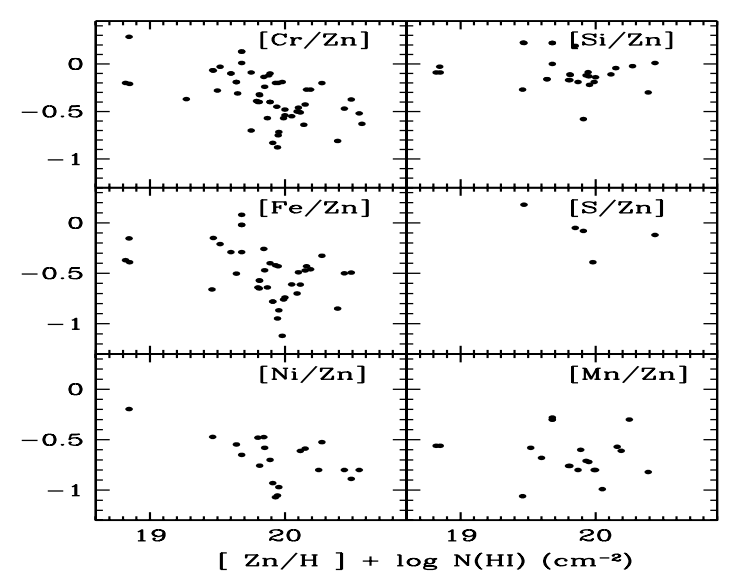

Figure 9. Abundance ratios $[\mathrm{M} / \mathrm{Zn}]$ in DLAs as a function of (a measure of) $\mathrm{Zn}$ column density $\mathcal{F}=[Z n / H]+\log (N H I)$. On the left: refractory elements have a declining $[\mathrm{M} / \mathrm{Zn}]$ with $[\mathrm{Zn} / \mathrm{H}]+\log (\mathrm{NHI})$; on the right: mildly refractory elements show no such trend.

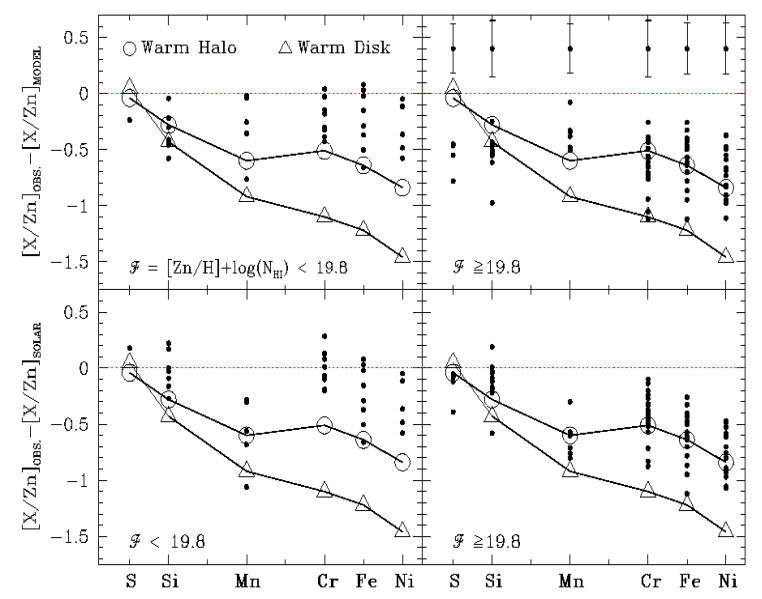

Figure 10. Depletion patterns in DLAs: abundance ratios $[\mathrm{X} / \mathrm{Zn}]$ are compared to model results (upper panels) and to solar composition (lower panels) for low Zn column densities (left) and high ones(right). The resulting depletion pattern ressembles the one of warm MW halo clouds (from Hou et al. 2001).

to relatively high redshifts (up to $z \sim 5$ ). Their study constitutes a powerful means to investigate the properties of distant galaxies (or of their building blocks). In particular, DLA metal abundances have been widely used in the past few years to probe the nature of DLAs (e.g. Prochaska \& Wolfe 2002 and references therein). However, it is not clear whether the observed abundances allow that, because of various biases: depletion of metals into dust (Pei and Fall 1995) or bias against too high or too low metal column densities (Boissé et al. 1998). The latter bias, in particular, may explain the observed absence of evolution in the absolute abundance of $\mathrm{Zn} / \mathrm{H}$ as a function of redshift, as suggested in Prantzos and Boissier (2000).

Abundance ratios offer a better diagnostic tool than absolute abundances for the study of the chemical evolution of a system. However, in the case of DLAs, the possibility of depletion into dust grains (even to a small extent) makes difficult a direct interpretation of the observed abundance patterns (see Vladilo 2002).

Hou et al. (2001) found an anticorrelation between the observed abundance ratio X/Zn (where $\mathrm{Zn}$ is assumed to be undepleted and $\mathrm{X}$ stands for the refractories $\mathrm{Fe}, \mathrm{Cr}$ and $\mathrm{Ni}$ ) and metal column density (Fig. 9). They suggested that this trend is an unambiguous sign of depletion, since metal column density is a measure of the amount of dust along the line of sight. Prochaska and Wolfe (2002) reached similar conclusions, finding that the anticorrelation is also present if $\mathrm{S}$ is used instead of $\mathrm{Zn}$.

Once dust depletion is unambiguously found, it is interesting to see whether the depletion pattern ressembles to the ones encountered in the various gaseous phases of the Milky 
Way (cold or warm clouds of the halo or the disk, see Savage and Sembach 1996). Hou et al. (2001) found that : if one assumes that the intrinsic metallicity pattern in DLAs is the one given by their models (of disk galaxies), a difficulty arises with $\mathrm{S}$, which is found to be depleted at high $\mathcal{F}=[Z n / H]+\log (N H I)$; if, on the other hand, it is assumed that the intrinsic DLA pattern is solar, a problem arises with $\mathrm{Mn}$, which is found to be depleted at low $\mathcal{F}$. In both cases the depletion patterns ressemble more to the one of warm halo clouds in the Milky Way (Fig. 10).

In any case, further studies of abundance patterns in DLAs will allow to probe the nature of those systems and to study the early phases of cosmic chemical evolution.

\section{REFERENCES}

1. Andrievsky S., Kovtyukh V., Luck R. et al. (2002a), A\&A 381, 32

2. Andrievsky S., Bersier D., Kovtyukh V. et al. (2002b), A\&A 384, 140

3. Argast D., Samland M., Gerhard O., Thielemann F.-K. (2000), A\&A 356, 873

4. Argast D., Samland M., Thielemann F.-K., Gerhard O. (2002), A\&A 388, 842

5. Boissé P., Le Brun V., Bergeron J., Deharveng J. (1998), A\&A 333,841

6. Boissier S., Prantzos N. (1999), MNRAS 307, 857

7. Carretta E., Gratton R., Cohen J. et al. (2002), astro-ph/0204083

8. Cunha K., Daflon S. (2002), in CNO in the Universe, Eds. G. Meynet et al., in press

9. Deharveng L., Pena M., Caplan J., Costero R. (2000), MNRAS 311, 329

10. Gay P., Lambert D. (2000), ApJ 533, 260

11. Goswami A., Prantzos N. (2000), A\&A 359, 191

12. Hou, J., Prantzos N., Boissier S. (2000), A\&A 362, 921

13. Hou, J., Boissier S., Prantzos N. (2001), A\&A 370, 23

14. Ishimaru Y., Prantzos N., Wanajo S. (2002) in preparation

15. Iwamoto K., Braschwitz F., Nomoto K., et al. (1999), ApJS 125, 439

16. Karlsson T., Gustafsson B. (2001), A\&A 379, 461

17. Kroupa P., Tout C., Gilmore G. (1993), MNRAS 262, 545

18. Limongi M., Straniero O., Chieffi A. (2000), ApJS, 129, 625

19. Maciel W., Da Costa R., 2001, astro-ph/0112210

20. Meynet G., Maeder A. (2002), A\&A 390, 561

21. Pei Y., Fall M. (1995), ApJ 454, 69

22. Pilyugin L., Ferrini F, Shkvarum R. (2002) in CNO in the Universe, Eds. G. Meynet et al., in press

23. Prantzos N., Boissier S. (2000), MNRAS 313, 338

24. Prochaska J., Wolfe A. (2002), ApJ 566, 68

25. Ryan S., Norris J, Beers T. (1996), ApJ 471, 254

26. Savage B., Sembach K. (1996), ARA\&A 34, 279

27. Timmes F., Woosley S., Weaver T. (1995), ApJS 98, 617

28. Truran J., Cowan J., Pilachowski A., Sneden C. (2002), astro-ph/0209308

29. Tsujimoto T., Shigeyama T. (1998) ApJL 508, 151

30. van den Hoek L., Groenewegen M. (1997), A\&AS, 123, 305

31. Vladilo G. (2002), ApJ 569, 295

32. Woosley S., Weaver T. (1995), ApJS 101, 823 